\title{
Aproximación diagnóstica ultrasonográfica prenatal al síndrome de Omenn: reporte de caso
}

\section{Prenatal ulltrasound diagnostic approach to Omenn} syndirome: case report

Santiago Aristizábal-Ortiz, $M D^{1}$; Alba Esquivel-Villabona, $M D, M S c^{2}$; Yenny Constanza

Bernal-Cifuentes, $M D^{3}$

Recibido: 24 de marzo de 2021/Aceptado: 4 de agosto de 2021

\section{RESUMEN}

Objetivo: reportar el caso de una gestante con diagnóstico ultrasonográfico de alteración del sistema tegumentario fetal y diagnóstico posnatal de síndrome de Omenn.

Presentación de caso: se presenta el caso de paciente de 27 años con embarazo de 31 semanas, con exploración ultrasonográfica prenatal que evidencia feto con edema significativo del cuero cabelludo, líquido amniótico ecogénico y descamación de la piel abdominal, con aproximación diagnóstica ultrasonográfica de variante de ictiosis. El recién nacido presentó eritrodermia congénita complicada con infección de piel, con posterior choque séptico y muerte. El estudio genético y patológico concluye síndrome de Omenn.

Conclusión: el síndrome de Omenn debe ser tomado en cuenta en los diagnósticos diferenciales cuando en la ultrasonografía prenatal existan hallazgos ultrasonográficos de una alteración del sistema tegumentario. Se requieren estudios que evalúen la

* Correspondencia: Santiago Aristizábal - saristizabal@unbosque.edu.co Av. Cra. 9 No. 131A-02, Universidad el Bosque, Bogotá (Colombia).

1. Residente de Radiología e Imágenes Diagnósticas, Fundación Santa Fe de Bogotá (Colombia).

2. Ginecóloga y Obstetra, Medicina Materno-fetal, Fundación Santa Fe de Bogotá. Docente Universidad El Bosque y Universidad de los Andes, Bogotá (Colombia).

3. Residente de Ginecología y Obstetricia, Universidad El Bosque, Bogotá (Colombia). exactitud del ultrasonido en el diagnóstico antenatal de las eritrodermias.

Palabras clave: inmunodeficiencia combinada grave, síndrome de Omenn, ictiosis, ultrasonografía prenatal.

\section{ABSTRACT}

Objective: To report the case of a pregnant woman with ultrasound diagnosis of altered fetal tegumental system and postnatal diagnosis of Omenn syndrome. Case presentation: A 27-year-old patient who presented at 31 weeks of gestation with prenatal ultrasound evidence of a fetus with significant scalp edema, echogenic amniotic fluid and scaly abdominal skin, with ichtyosis variant impression on diagnostic ultrasound. The baby was born with congenital erythroderma complicated with skin infection, and later developed septic shock and died. The genetic and pathologic workup led to the conclusion of Omenn syndrome.

Conclusion: Omenn syndrome must be considered as part of the differential diagnoses when prenatal ultrasound shows findings of altered tegument system. Studies are required to assess the accuracy of ultrasound for prenatal diagnosis of erythroderma.

Keywords: Severe combined immunodeficiency, Omenn syndrome, ichthyosis, fetal ultrasonography. 


\section{INTRODUCCIÓN}

Las eritrodermias neonatales congénitas son un grupo heterogéneo de desórdenes donde existe eritema persistente de la piel que involucra más del $90 \%$ de la superficie corporal (1). Entre las etiologías más comunes se deben tener en cuenta los desórdenes de la cornificación (incluyendo las variantes de ictiosis) y los desórdenes inflamatorios (dermatitis atópica, psoriasis, dermatitis seborreica, entre otros); además, los trastornos metabólicos como la deficiencia de biotinidasa y de zinc, y los inmunológicos como la inmunodeficiencia combinada grave y el síndrome de Omenn, aunque menos frecuentes, deben ser considerados como diagnósticos diferenciales (2).

El síndrome de Omenn, también conocido como inmunodeficiencia combinada con hipereosinofilia, fue descrito en 1965 por Gilbert Omenn (3), es una enfermedad autosómica recesiva, extremadamente rara, con una incidencia de 1:5.000.000 y una relación hombre-mujer de 1:1 (4). Se caracteriza por presentar los síntomas de la inmunodeficiencia combinada grave, asociados a eritrodermia, alopecia, linfadenopatía, hepatoesplenomegalia e inflamación de distintos órganos (enteritis, pneumonitis o hepatitis), y se muestra con un inmunofenotipo característico de linfocitosis $\mathrm{T}$, ausencia de linfocitos $\mathrm{B}$, ausencia de marcadores de timopoyesis, alteración de la proliferación de linfocitos $\mathrm{T}$ frente a la estimulación con fitohemaglutinina, ausencia de respuesta inmunológica a los patógenos de las vacunas y aumento de los valores séricos de la inmunoglobulina $\mathrm{E}$, lo que genera un estado proinflamatorio sistémico persistente y una susceptibilidad a infecciones por patógenos oportunistas (5). Desde el punto de vista genético, en la mayoría de los casos existen mutaciones de patrón autosómico recesivo de genes que codifican para las enzimas RAG1 y RAG2 (recombination-activating genes, en inglés), las cuales son exclusivamente expresadas por linfocitos inmaduros y tienen como función ser catalizadores de la recombinación $\mathrm{V}(\mathrm{D}) \mathrm{J}$ (variable[diversity] Joining en inglés), la cual es fundamental para la diversidad de inmunoglobulinas y receptores de células T (6). No obstante, existen numerosas descripciones en la literatura que asocian este síndrome con mutaciones por otros genes, entre estos el gen responsable de la expresión de la proteína Artemis, la cual facilita la apertura de horquillas de ADN en extremos codificadores (7), el gen RPMRP RNA (8) y el gen IL7RA (9).

Los avances en ultrasonido 2D y 3D permiten, hoy en día, la evaluación detallada de estructuras del sistema tegumentario y de la extensión lateral y axial de múltiples lesiones en el paciente con psoriasis (10). Este uso del ultrasonido abre la posibilidad de aplicarlo en el diagnóstico prenatal de las alteraciones congénitas que afectan la piel del neonato, entre ellas la eritrodermia congénita, principalmente la ictiosis y el síndrome de Omenn.

Con la intención de aportar información sobre esta nueva aplicación del ultrasonido presentamos el reporte de caso de un recién nacido con eritodermia y diagnóstico final de síndrome de Omenn, en el cual la aproximación diagnóstica ultrasonográfica antenatal se inclinó por alguna variante de ictiosis de características leves. Se debe señalar que en el síndrome de Omenn, el diagnóstico prenatal temprano tiene implicaciones terapéuticas importantes que pueden impactar favorablemente la calidad de vida y el pronóstico de los pacientes con esta condición (11).

\section{PRESENTACIÓN DE CASO}

Se presenta el caso de una paciente de 27 años, primigestante, con embarazo de 33,4 semanas que, por cuadro de actividad uterina irregular, consulta por urgencias a una institución general privada de alta complejidad, que atiende pacientes del régimen contributivo de los trabajadores y las empresas de aseguramiento privadas en el Sistema General de Seguridad Social en Colombia. La paciente no tenía antecedentes patológicos o de consanguinidad importantes, enfermedades genéticas o antecedentes familiares de mortalidad neonatal temprana. Al examen físico se encontró útero grávido con feto único cefálico, frecuencia cardiaca fetal de 155 latidos por minuto, y actividad uterina regular de gran intensidad. Al tacto vaginal se detectó cuello corto, blando, posterior, sin evidencia de sangrado o amniorrea. Se inició manejo tocolítico con nifedipina, dosis de crisis de $10 \mathrm{mg}$ vía oral cada $20 \mathrm{~min}$ y luego $10 \mathrm{mg}$ vía oral cada 8 h; analgésico, con acetaminofén 1000 
mg vía oral cada 6 h más bromuro de hioscina 20 mg endovenoso cada 8 h, y esquema de maduración pulmonar con betametasona intramuscular, $12 \mathrm{mg}$ cada $24 \mathrm{~h}$, dos dosis. Los laboratorios de ingreso reportaron leucocitos 16100/uL con granulocitos $83 \%$, hemoglobina 13,8 g/dL, plaquetas 274000/ uL, proteína C reactiva de $1,12 \mathrm{mg} / \mathrm{dL}$ y parcial de orina límpido sin leucocitos ni nitritos con $\mathrm{pH}$ de 7. Se realizó ultrasonido obstétrico que evidenció crecimiento fetal acorde con peso fetal estimado de $2.327 \mathrm{~g}$ en percentil 51 y circunferencia abdominal mayor que el percentil 97 secundaria a edema. Líquido amniótico con aumento de su ecogenicidad, de características particuladas, con índice de líquido amniótico (ILA) de $28 \mathrm{~cm}$, ausencia de visualización de cámara gástrica, edema de piel en extremidades; a la visualización del rostro en 3D se aprecian imágenes en copo de nieve (Figura 1), con edema predominante en la región frontal (Figura 2). Con estos hallazgos se considera como primera posibilidad diagnóstica una eritrodermia congénita de características leves, más alguna variante de ictiosis.

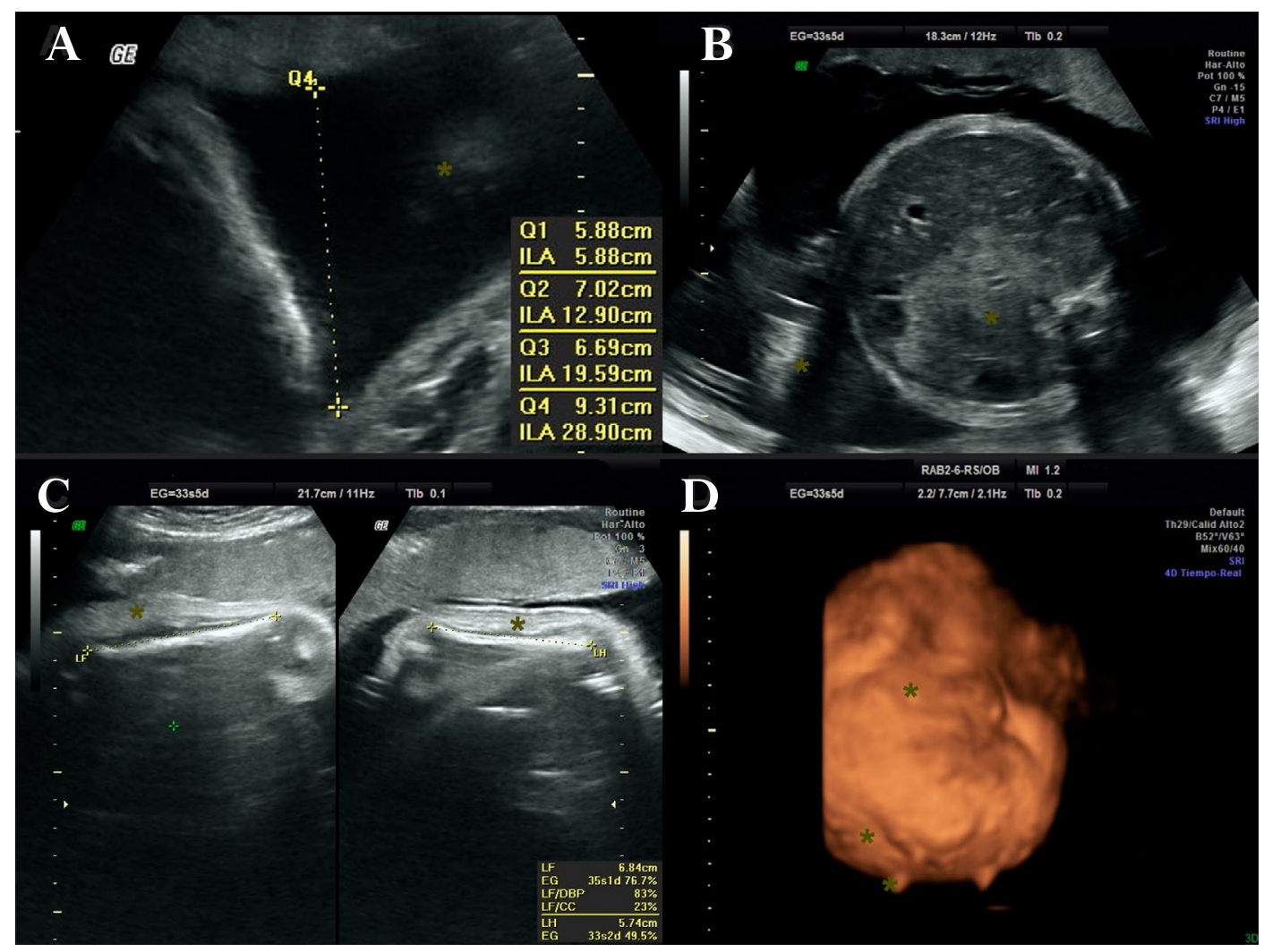

Figura 1. Hallazgos en ultrasonido de alteraciones tegumentarias en feto con síndrome de Omenn.

(A) Polihidramnios y líquido amniótico con sedimento. (B) Ausencia de visualización de cámara gástrica. (C) Edema de piel en extremidades. (D) Piel con imagen en copo de nieve a la exploración tridimensional del rostro.

La gestante es remitida a otra institución de cuarto nivel de complejidad por disposición del tercer pagador (aseguradora). Debido a los hallazgos ultrasonográficos es hospitalizada y tres días después presenta ruptura espontánea de membranas con líquido meconial, por lo cual es llevada a cesárea de urgencia; se obtiene recién nacida viva pretérmino de 34 semanas, con peso en percentil 50 y adaptación espontánea. Al examen físico se evidencian lesiones descamativas y eritematosas en piel en el $100 \%$ de la superficie corporal total y facies dismórficas (Figura 2). La recién nacida presenta taquipnea transitoria del recién nacido y se inicia oxigenoterapia. Por las características ecográficas y los hallazgos en piel se considera como primera posibilidad diagnóstica ictiosis laminar y se ingresa a la unidad de cuidados intensivo neonatal (UCIN), donde se inicia manejo médico. Posteriormente, presentó una evolución 
tórpida con choque séptico (foco primario piel), por lo que es evaluada por especialistas en neonatología, infectología, nefrología y cirugía plástica. Se inició terapia antibiótica de amplio espectro y se ajustó posteriormente con el reporte de hemocultivos con crecimiento de Pseudomonas aeruginosa y Enterococcus faecium. La infección progresó a falla multiorgánica, con disfunción ventilatoria, insuficiencia renal aguda, coagulación intravascular diseminada, trombocitopenia severa secundaria y anemia severa multifactorial que requirió de terapia de reemplazo renal, manejo inotrópico múltiple, politransfusiones de plaquetas y plasma, y transfusiones de glóbulos rojos. Es llevada a procedimiento quirúrgico por parte de cirugía plástica realizando desbridamiento de las lesiones y colocación de amnios inicialmente en tórax, las lesiones se describen como piel escaldada con exposición de la dermis superficial en el $100 \%$ de la superficie corporal. La recién nacida falleció a los 18 días de vida.

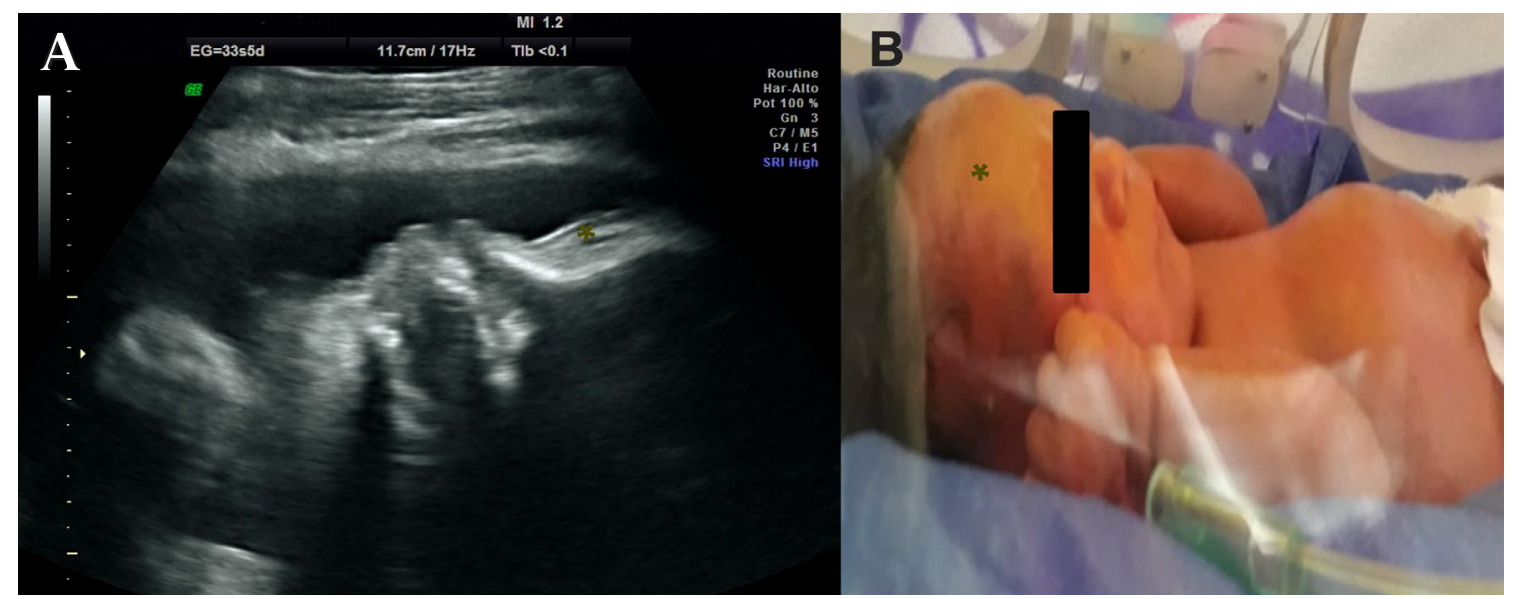

Figura 2. Correlación de hallazgos por ultrasonido en rostro de feto con síndrome de Omenn. (A) y (B) Piel con edema y descamación.

Previa autorización de los padres, se realizó autopsia clínica en la recién nacida, en la cual se reportó esfacelación en el $90 \%$ de la superficie corporal y alopecia en cejas y pestañas. En el tejido tegumentario se evidencian extensas áreas con pérdida de la epidermis e hipogranulosis, y colonización del tejido por cándida. Cerebro con parénquima inmaduro y hemorragia intraventricular, sistema linforreticular y hematopoyético con múltiples zonas de necrosis y descenso de la población linfoide; a nivel del bazo y timo se encuentra disminución del tejido linfoide. Estos hallazgos sugirieron síndrome de Omenn como primera posibilidad diagnóstica. Teniendo en cuenta la sospecha diagnóstica patológica, y al ser el primer hijo de la pareja, se decide realizar estudio genético complementario. Se realiza secuenciación genética de segunda generación, en la cual se identifica duplicación heterocigota, probablemente patogénica, de una timina en la posición 829 de ADNc del gen RAG2 (c.829dupT).

Teniendo en cuenta los resultados de la necropsia y la variante probablemente patogénica del gen RAG2 se concluye el diagnóstico de síndrome de Omenn.

Aspectos éticos. Este reporte de caso contó con el consentimiento de ambos padres de la recién nacida, y siguió todos los protocolos en investigación médicocientífica de la institución base, la cual permanece anónima por petición de los padres.

\section{DISCUSIÓN}

En este reporte de caso presentamos los hallazgos antenatales por ultrasonido obstétrico de un recién nacido con eritrodermia congénita y diagnóstico final de síndrome de Omenn. Después de una búsqueda de la literatura solo identificamos un reporte de caso en un recién nacido con esta condición (12). 
En este reporte describen los siguientes hallazgos ultrasonográficos: edema pronunciado del cuero cabelludo, líquido amniótico ecogénico, desprendimiento de la piel abdominal y presencia de detritos intraabdominales y polihidramnios.

El diagnóstico diferencial más importante es la ictiosis congénita, de la cual identificamos nueve reportes de caso. En estos, se informan como hallazgos ultrasonográficos importantes las alteraciones de líquido amniótico (líquido amniótico ecogénico, oligo o polihidramnios) (13-18), el edema de la piel (14), los detritos intrabdominales (17), la presencia de malformaciones morfológicas faciales de las cuales se describen edema de los labios y boca grande $(13,14,16,18-20)$, la hipoplasia nasal o perfil facial plano $(13-16,18,20,21)$ y las malformaciones auriculares $(13-16,20)$; también se describe la flexión fija de manos y pies u otras malformaciones de la extremidades (13-16,18-20). De estas características ultrasonográficas, solo las alteraciones de líquido amniótico (particularmente el polihidramnios y el líquido amniótico ecogénico), los detritos intraabdominales y el edema de la piel (específicamente en el cuero cabelludo) han sido descritas en el síndrome de Omenn (12).

Consideramos, al igual que Bharti y Gibson, que el ultrasonido podría ser un aliado importante en la identificación prenatal y, por lo tanto, en el diagnóstico temprano del síndrome de Omenn, y que este debe ser tomado en cuenta como diagnóstico diferencial cuando existan hallazgos morfológicos ultrasonográficos antenatales que sugieran algún tipo de alteración del sistema tegumentario (12). Surge la hipótesis de que si se trata de una primera gestación y se encuentran estos hallazgos ultrasonográficos fetales, sin antecedentes familiares de consanguinidad, sin alteraciones genéticas familiares ni mortalidades neonatales previas, se debe considerar la posibilidad de una inmunodeficiencia primaria y estarían indicados los exámenes genéticos en el feto; entre ellos el tamizaje neonatal con conteo de linfocitos, la reacción en cadena de polimerasa (PCR) cuantitativa para círculos de escisión del receptor de las células T (TREC) y el ensayo por inmunoabsorción ligado a enzimas (ELISA), especialmente para interleuquina 7 (IL-7) (11). Por el contrario, si se identifican hallazgos del sistema tegumentario fetal, asociados o no a alteraciones morfológicas faciales o de extremidades, y se trata de una gestante con antecedentes familiares genéticos o gestaciones previas con hallazgos de ictiosis y mortalidad preconcepcional o neonatal temprana, se debe realizar el estudio genético prenatal en búsqueda de alguna variante de ictiosis. En las parejas que tienen este antecedente se debe considerar la consulta preconcepcional como herramienta de decisión para futuras gestaciones (4).

El síndrome de Omenn, aunque de baja incidencia, tiene implicaciones terapéuticas muy diferentes a la ictiosis y, en general, a cualquier otra eritrodermia neonatal, ya que se ha descrito que los pacientes que reciben un trasplante de médula ósea temprano tienen mejores desenlaces que aquellos que lo reciben de forma tardía (11).

No obstante, este reporte de caso tiene algunas limitaciones respecto al diagnóstico definitivo del síndrome de Omenn. Aunque el diagnóstico se basó en el examen físico posnatal y los hallazgos patológicos de la necropsia típicos reportados en la literatura para este síndrome, el estudio de secuenciación genética mostró solo una variante heterocigótica, posiblemente patogénica, del gen RAG2 que, por no encontrarse en forma homocigota o heterocigota compuesta, no puede confirmar un diagnóstico genético definitivo; no obstante, las mutaciones tipo frameshift, que generan pérdida de función, son un mecanismo conocido de patogenicidad de este gen (22). Más aún, los hallazgos ultrasonográficos, asociados al examen físico posnatal, y los hallazgos patológicos de la necropsia típicos reportados en la literatura para el síndrome de Omenn apoyan el diagnóstico. Como complemento, se requeriría un análisis de deleciones/duplicaciones del gen RAG2 para evaluar otra posible variante de este tipo en otro alelo del gen, que podría esclarecer la etiopatogenia genética precisa. 


\section{CONCLUSIONES}

Ante los hallazgos morfológicos de alteraciones tegumentarias en el ultrasonido fetal que sugieran algún tipo de alteración del sistema tegumentario (especialmente ictiosis) se debe considerar el diagnóstico de síndrome de Omenn dado el posible beneficio antenatal. Es necesario determinar la utilidad del ultrasonido en el diagnóstico prenatal de las eritrodermias ya que esta no ha sido establecida ni correlacionada con los patrones de otros diagnósticos genéticos e histopatológicos.

\section{REFERENCIAS}

1. Sarkar R, Garg S, Garg V. Neonatal erythroderma (red baby). Indian J Paediatr Dermatol. 2013;14(3):47. https://doi.org/10.4103/2319-7250.122160

2. Boull CL, Hook K. Neonatal erythroderma - Clinical perspectives. RRN. 2017;7:1-9. https://doi. org/10.2147/RRN.S104667

3. Omenn GS. Familial reticuloendotheliosis with eosinophilia. N Engl J Med. 1965;273(8):427-32. https:// doi.org/10.1056/NEJM196508192730806

4. Hsu CC, Lee JYY, Chao SC. Omenn syndrome: A case report and review of literature. Dermatologica Sin. 2011;29(2):50-4. https://doi.org/10.1016/j. dsi.2011.05.002

5. Gennery AR. Advances in genetic and molecular understanding of Omenn syndrome - Implications for the future. Expert Opinion Orphan Drugs. 20183;6(6):3519. https://doi.org/10.1080/21678707.2018.1478287

6. Corneo B, Moshous D, Güngör T, Wulffraat N, Philippet $\mathrm{P}$, Deist FL, et al. Identical mutations in RAG1 or RAG2 genes leading to defective $\mathrm{V}(\mathrm{D}) \mathrm{J}$ recombinase activity can cause either T-B-severe combined immune deficiency or Omenn syndrome. Blood. 2001;97(9):2772-6. https:// doi.org/10.1182/blood.V97.9.2772

7. Ege M. Omenn syndrome due to ARTEMIS mutations. Blood. 2005;105(11):4179-86. https://doi. org/10.1182/blood-2004-12-4861

8. Roifman C, Gu Y, Cohen A. Mutations in the RNA component of RNase mitochondrial RNA processing might cause Omenn syndrome. J Allergy Clin Immunol. 2006;117(4):897-903. https://doi.org/10.1016/j. jaci.2006.01.003
9. Giliani S, Bonfim C, de Saint Basile G, Lanzi G, Brousse N, Koliski A, et al. Omenn syndrome in an infant with IL7RA gene mutation. J Pediatr. 2006;148(2):272-4. https://doi.org/10.1016/j.jpeds.2005.10.004

10. Crisan M, Badea R, Crisan D, Bezugly A, Colosi H, Strilciuc $S$, et al. Ultrasonography as a new, noninvasive imagistic technique used for the diagnosis and monitoring of psoriasis. In: Chiriac A, editor. An Interdisciplinary Approach to Psoriasis. InTech; 2017. https://doi.org/10.5772/67812

11. Lipstein EA, Vorono S, Browning MF, Green NS, Kemper AR, Knapp AA, et al. Systematic evidence review of newborn screening and treatment of severe combined immunodeficiency. Pediatrics. 20101;125(5):e122635. https://doi.org/10.1542/peds.2009-1567

12. Bharti D, Gibson J. Fetal sonographic features in an infant with Omenn syndrome. J Pregnancy Newborncare. $2018 ; 1(2): 3$.

13. Bongain A, Benoit B, Ejnes L, Lambert JC, Gillet JY. Harlequin fetus: Three-dimensional sonographic findings and new diagnostic approach. Ultrasound Obstet Gynecol. 2002;20(1):82-5. https://doi. org/10.1046/j.1469-0705.2002.00708.x

14. Berg C, Geipel A, Kohl M, Krokowski M, Baschat A, Germer U, et al. Prenatal sonographic features of Harlequin ichthyosis. Arch Gynecol Obstet. 2003;268(1):48-51. https://doi.org/10.1007/s00404002-0333-4

15. Holden S, Ahuja S, Ogilvy-Stuart A, Firth HV, Lees C. Prenatal diagnosis of Harlequin ichthyosis presenting as distal arthrogryposis using three-dimensional ultrasound. Prenat Diagn. 2007;27(6):566-7. https:// doi.org/10.1002/pd.1727

16. Kudla MJ, Timmerman D. Prenatal diagnosis of harlequin ichthyosis using 3- and 4-dimensional sonography. J Ultrasound Med. 2010;29(2):317-9. https:// doi.org/10.7863/jum.2010.29.2.317

17. Blaas HGK, Salvesen KA, Khnykin D, Jahnsen FL, Eik-Nes SH. Prenatal sonographic assessment and perinatal course of ichthyosis prematurity syndrome: Perinatal course of ichthyosis prematurity syndrome. Ultrasound Obstet Gynecol. 2012;39(4):473-7. https://doi.org/10.1002/uog.9014

18. Tourette C, Tron E, Mallet S, Levy-Mozziconacci A, Bonnefont JP, D'Ercole C, et al. Three-dimensional 
ultrasound prenatal diagnosis of congenital ichthyosis: Contribution of molecular biology. Prenat Diagn. 2012;32(5):498-500. https://doi.org/10.1002/ pd.3839

19. Watson WJ, Mabee LM. Prenatal diagnosis of severe congenital ichthyosis (harlequin fetus) by ultrasonography. J Ultrasound Med. 1995;14(3):241-3. https:// doi.org/10.7863/jum.1995.14.3.241

20. Jian W, Du QT, Lai ZF, Li YF, Li SQ, Xiong ZT, et al. Prenatal diagnose of a fetus with Harlequin ichthyosis in a Chinese family. Taiwan J Obstet Gynecol. 2018;57(3):452-5. https://doi.org/10.1016/j. tjog.2018.04.023

21. Meizner I. Prenatal ultrasonic features in a rare case of congenital ichthyosis (harlequin fetus). J Clin Ultrasound. 1992;20(2):132-4. https://doi.org/10.1002/ jcu. 1870200209

22. Notarangelo LD, Kim M-S, Walter JE, Lee YN. Human RAG mutations: Biochemistry and clinical implications. Nat Rev Immunol. 2016;16(4):234-46. https:// doi.org/10.1038/nri.2016.28

\section{FINANCIACIÓN}

Este proyecto no tuvo fuentes de financiación.

\section{CONTRIBUCIÓN DE LOS AUTORES}

Santiago Aristizábal-Ortiz: concepción y diseño; adquisición de datos; análisis e interpretación de datos; redacción del artículo; revisión crítica del artículo para contenido intelectual importante; participación suficiente en el trabajo para asumir la responsabilidad del contenido.

Alba Esquivel-Villabona: concepción y diseño; adquisición de datos; análisis e interpretación de datos; revisión crítica del artículo para contenido intelectual importante; participación suficiente en el trabajo para asumir la responsabilidad del contenido; aprobación final de la versión publicada.

Yenny Constanza Bernal-Cifuentes: adquisición de datos y redacción del artículo.

\section{Conflicto de intereses: ninguno declarado.}

